\title{
Propagating compaction bands in confined compression of snow
}

\author{
Thomas W. Barraclough', Jane R. Blackford', Stefan Liebenstein ${ }^{1,2}$, Stefan Sandfeld ${ }^{2}$, Tim J. Stratford', \\ Gerhard Weinländer ${ }^{2}$ and Michael Zaiser ${ }^{1,2 \star}$
}

Some materials are strong in response to a slowly applied deformation, yet weak when subject to rapid deformations-a materials property known as strain-rate softening'. Snow exhibits such behaviour: it is comparatively strong at low deformation rates, where it is quasi-plastic, but weak at high rates, where it deforms in a quasi-brittle manner ${ }^{2}$. During deformation, strain-rate-softening materials ranging from metals $s^{3,4}$ to micellar systems ${ }^{5}$ exhibit complex spatio-temporal deformation patterns, including regular or chaotic deformation-rate oscillations and travelling deformation waves ${ }^{6}$. Here we report a systematic investigation of such phenomena in snow and show that snow can deform with the formation and propagation of localized deformation bands accompanied by oscillations of the driving force. We propose a model that accounts for these observations. Our findings demonstrate that in snow, strain localization can occur even in initially homogeneous samples deforming under homogeneous loads.

Irreversible deformation of snow plays an important role in a number of problems, ranging from the interaction of snow with winter sports equipment ${ }^{7}$, vehicle traction $^{8}$, to snowpack stability and avalanche release ${ }^{9}$. Snow strength depends strongly on cohesive bonds between ice granules in the snow microstructure. Shear deformation reduces snow strength as bonds are broken or sheared ${ }^{10}$, whereas formation of new cohesive bonds between ice granules - a thermodynamically driven sintering process ${ }^{11-13}$-leads to strengthening of snow over time (ageing) ${ }^{14}$. Rapid deformation leaves insufficient time for sintering to restore broken bonds; therefore, snow is at high rates of deformation weaker than at low rates. This goes along with a transition from quasi-plastic (low rates) to quasi-brittle deformation behaviour (high rates), which has been observed in shear ${ }^{14}$ as well as in compressive deformation ${ }^{15}$.

Here we report the results of confined compression experiments performed at strain rates intermediate between the quasi-plastic and quasi-brittle regimes. Specimens of both natural and artificially produced dry snow with densities $\rho$ between $275 \mathrm{~kg} \mathrm{~m}^{-3}$ and $370 \mathrm{~kg} \mathrm{~m}^{-3}$ and mean grain size $\xi \approx 0.2 \mathrm{~mm}$ were contained within a rectangular transparent container with aluminium alloy side walls and a glass front and back. After different waiting (ageing) times at a temperature of $-10^{\circ} \mathrm{C}$, the specimens were compacted by a piston moving at fixed rates ranging from $0.01 \mathrm{~mm} \mathrm{~s}^{-1}$ to $5 \mathrm{~mm} \mathrm{~s}^{-1}$, providing nominal strain rates from $\dot{e}_{\text {ext }}=5 \times 10^{-5} \mathrm{~s}^{-1}$ to $2.5 \times 10^{-2} \mathrm{~s}^{-1}$. The axial driving force acting on the specimens was recorded by load cells located both above the piston and between the specimens and the apparatus ground plate. A 18-megapixel camera recorded images of the specimens, which were illuminated from behind, at $0.25 \mathrm{~s}$ intervals. To quantitatively characterize the deformation patterns, digital image correlation (DIC) was used to obtain spatio-temporal displacement records from which local strain and strain-rate tensors were calculated. For details of the experimental set-up, specimen preparation, and data analysis, see Supplementary Methods 1.

Figure 1 shows an image taken from the record of a typical compression test of an artificial snow sample, together with the corresponding $\epsilon_{x x}(x, z)$ and strain rate $\dot{\epsilon}_{x x}(x, z)$ fields. The full record is shown in Supplementary Movie 1. Compaction proceeds in a strongly heterogeneous manner: a compacted region, visible as a darker area, is separated from an uncompacted region by a moving front where the strain rate concentrates. This front is perpendicular to the compression axis (in the figure, $x$ axis). We visualize band motion by spacetime plots where we average over the perpendicular $z$ coordinate and plot the averaged strain rate $\dot{e}_{\text {tot }}(x, t)=\left\langle\dot{\epsilon}_{x x}(x, z, t)\right\rangle_{z}$ in colourscale as a function of $x$ and $t$ (Figs 2 and 3 ). In these plots we use Lagrangian coordinates where $x$ denotes the position along the compression axis in the initial, undeformed configuration. The colour contrast demonstrates strong deformation localization. The moving deformation bands appear on the spacetime plots as inclined zones of high strain rate. The slope of inclination defines the band velocity $v_{\mathrm{B}}$ in the Lagrangian frame. The first band nucleates at the top of the sample and moves at constant (Lagrangian) speed downwards until it reaches the bottom of the sample, leaving the sample in an almost homogeneously deformed state with strain $e_{\mathrm{B}} \approx 0.2$ ('band strain'). The band is then reflected and moves upwards across the sample until it reaches the top. Repetition of this process leads to a bouncing motion of the locus of deformation (see Supplementary Movie 1). Oscillatory features are also manifest on the force versus time curves, as band nucleation is associated with an up-down oscillation of the driving force. Occasionally the bouncing pattern is interrupted, as seen in Fig. 2c and Supplementary Movie 2, where the first band gets stuck and deformation is accommodated by a second band nucleating at the bottom and moving upwards until it merges with the first, whence a new band nucleates at the top and the bouncing pattern is resumed.

Systematic investigation of a series of artificial snow samples of different age $t_{\mathrm{a}}$ ranging from $3 \times 10^{2} \mathrm{~s}$ to $3 \times 10^{5} \mathrm{~s}$ reveals that the stress required for initiation and propagation of the first deformation band and also the band strain increase with specimen age (Supplementary Fig. 6). Increasing the strain rate from $\dot{e}_{\text {ext }}=5.6 \times 10^{-3} \mathrm{~s}^{-1}$ to $\dot{e}_{\text {ext }}=2.5 \times 10^{-2} \mathrm{~s}^{-1}$ increases the band velocity proportionally, but leaves the overall deformation pattern unchanged. A decrease in strain rate to values of $\dot{e}_{\text {ext }}=5 \times 10^{-4} \mathrm{~s}^{-1}$ or less makes the deformation bands disappear: at low strain rates, band formation gives way to homogeneous plastic flow (Fig. 2a).

\footnotetext{
${ }^{1}$ School of Engineering, Institute for Materials and Processes, University of Edinburgh, The King's Buildings, Sanderson Building, Edinburgh EH9 3JL, UK. ${ }^{2}$ Department of Materials Science, WW8-Materials Simulation, Friedrich-Alexander University Erlanger-Nürnberg, Dr.-Mack-Strasse 77, 90762 Fürth, Germany. *e-mail: michael.zaiser@ww.uni-erlangen.de
} 

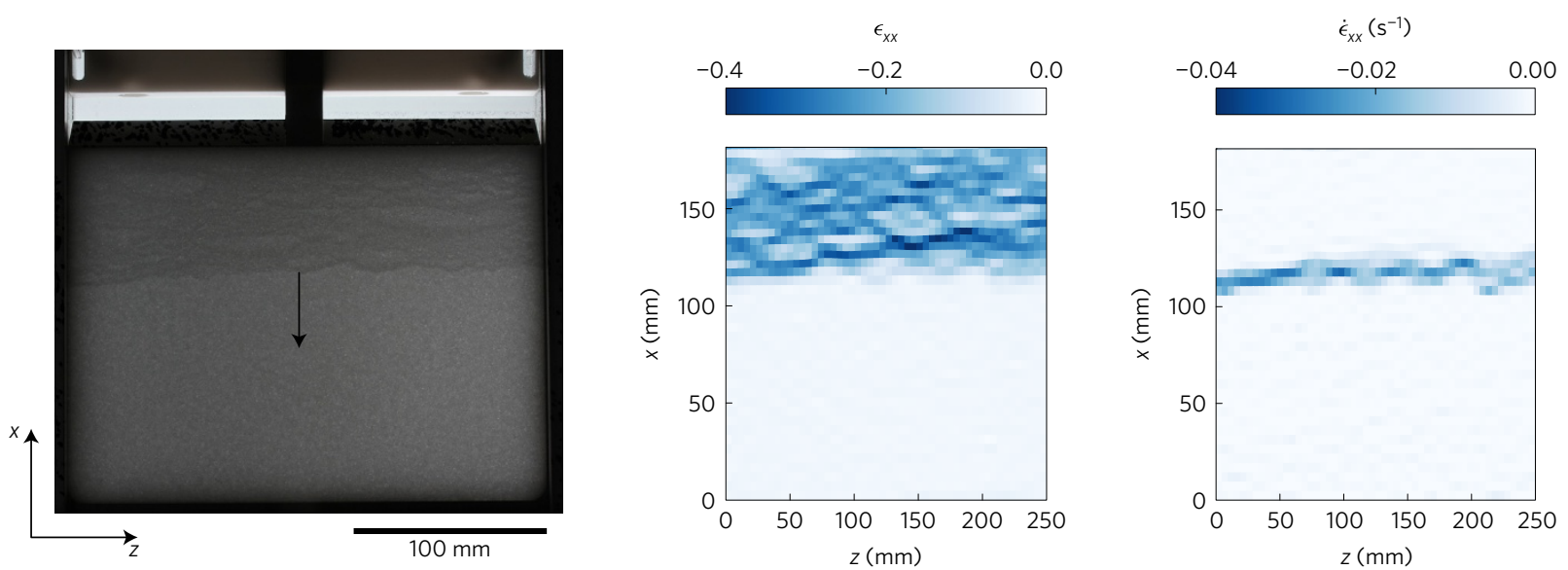

Figure 1 | Strain and strain-rate patterns in a laboratory-made snow specimen. Deformation rate $\dot{e}_{\mathrm{ext}}=5.6 \times 10^{-3} \mathrm{~s}^{-1}$, snapshot at $9.94 \%$ compressive strain. (Left) Photographic image showing the compaction front, the arrow indicates the front propagation direction; (centre) corresponding strain pattern as obtained by DIC; (right) corresponding strain-rate pattern. Note that the outermost edges of the sample are not covered by the DIC analysis.

a
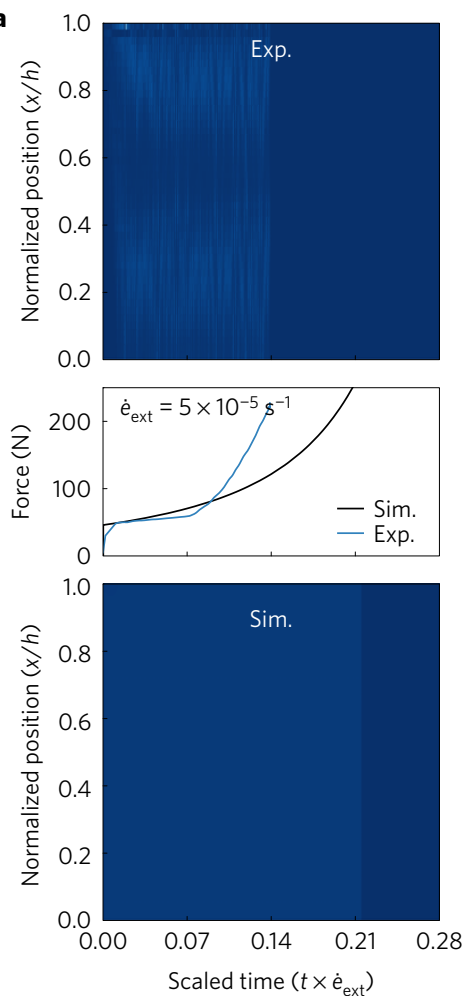
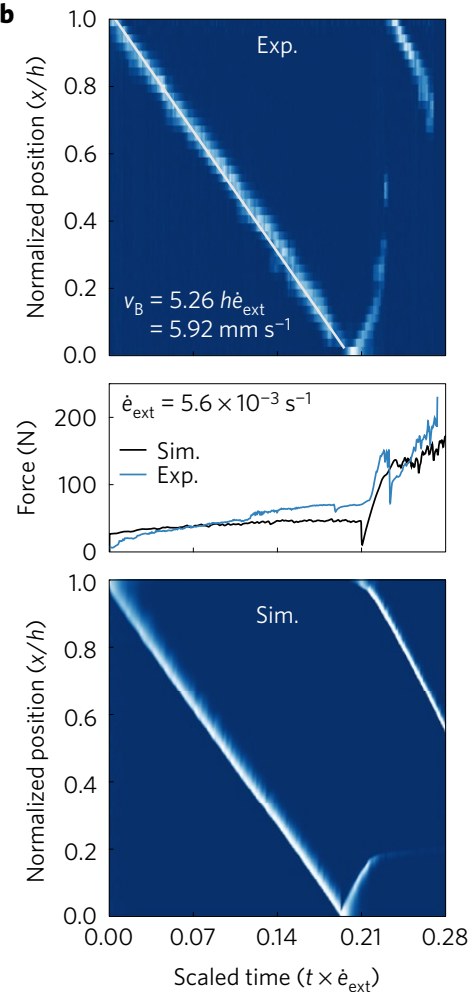
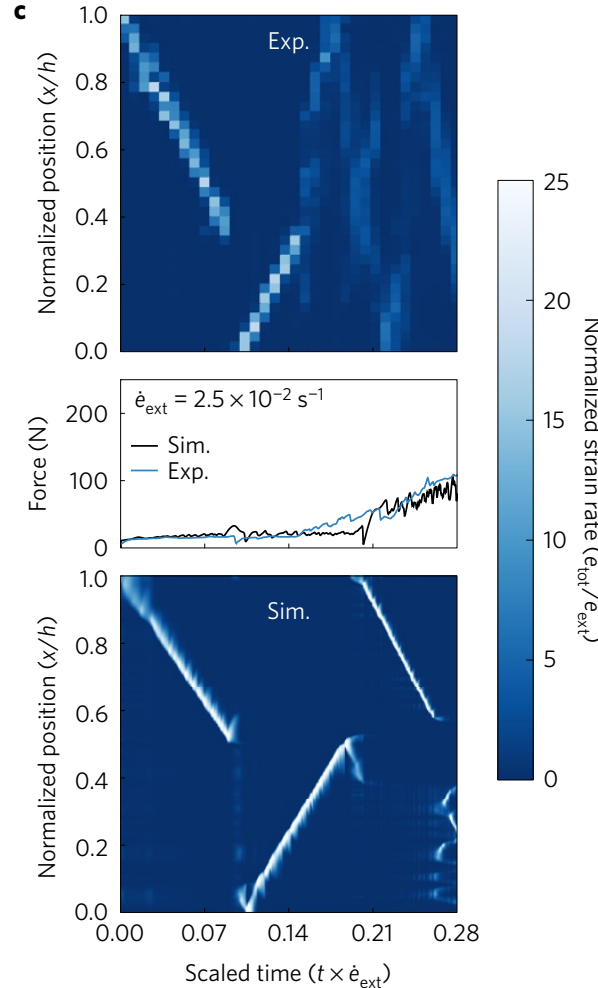

Figure 2 Spacetime plots of deformation activity in laboratory-made snow. Snow density $\rho=370 \mathrm{~kg} \mathrm{~m}^{-3}$, specimen age $t_{\mathrm{a}}=3 \times 10^{2} \mathrm{~s}$.

a, $\dot{e}_{\text {ext }}=5 \times 10^{-5} \mathrm{~s}^{-1} \cdot \mathbf{b}, \dot{e}_{\mathrm{ext}}=5.6 \times 10^{-3} \mathrm{~s}^{-1} \cdot \mathbf{c}, \dot{e}_{\mathrm{ext}}=2.5 \times 10^{-2} \mathrm{~s}^{-1}$. The top plots show the deformation activity as deduced experimentally from DIC, while the bottom plots show simulation data. The centre graphs are the corresponding force versus time/strain curves. Note that all three graphs in a column have the same $x$ axis.

Whereas artificial snow samples have the advantage of known specimen history and reproducible microstructure, it is essential to ascertain that natural snow shows similar behaviour. To this end we deformed natural snow samples harvested in the Cairngorm Mountains. The corresponding spacetime plots in Fig. 3 demonstrate the ubiquity of propagating compaction bands. Samples that were sawcut from a block of natural snow (Fig. 3a,b) show irregular band propagation patterns where bands often nucleate in the centre of the sample, and propagation is jerky with multiple band arrests and jumps of activity between different locations (Supplementary Movie 3). Nevertheless, the band strains $\varepsilon_{\mathrm{B}} \approx 0.2$ and band velocities $v_{\mathrm{B}}$ deduced from the spacetime plots compare well with those of artificial snow samples. Samples that were homogenized by sieving the snow into the sample container before the experiment deform differently (see Fig. 3c and Supplementary Movie 4); in such samples the band sequence is extremely regular, with bands bouncing repeatedly between the sample top and bottom, and the overall behaviour is similar to artificial snow.

The interplay between softening and ageing processes which makes snow a strain-rate-softening material has been modelled by a number of authors, for example, Louchet ${ }^{16}$ and Reiweger and colleagues ${ }^{17}$. However, these models cannot describe spatiotemporal strain localization because they do not account for the spatial structure of the deformation field. To model the observed 

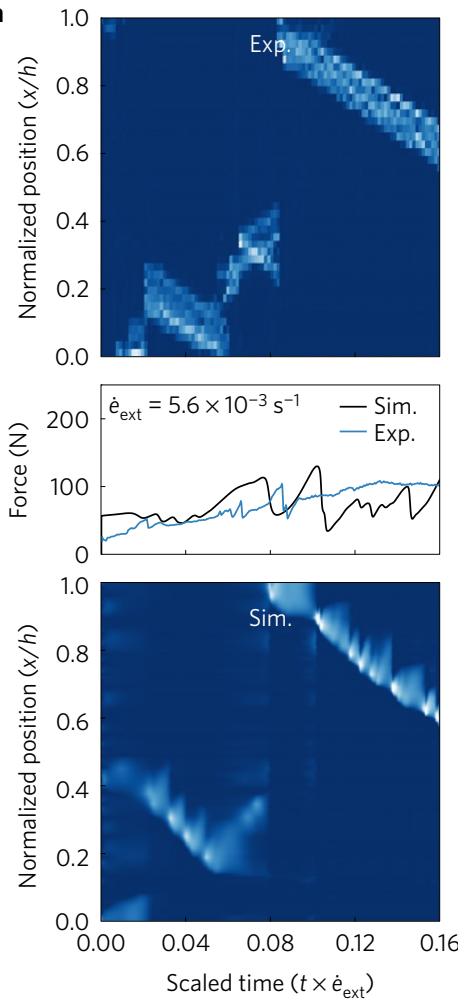

\section{b}
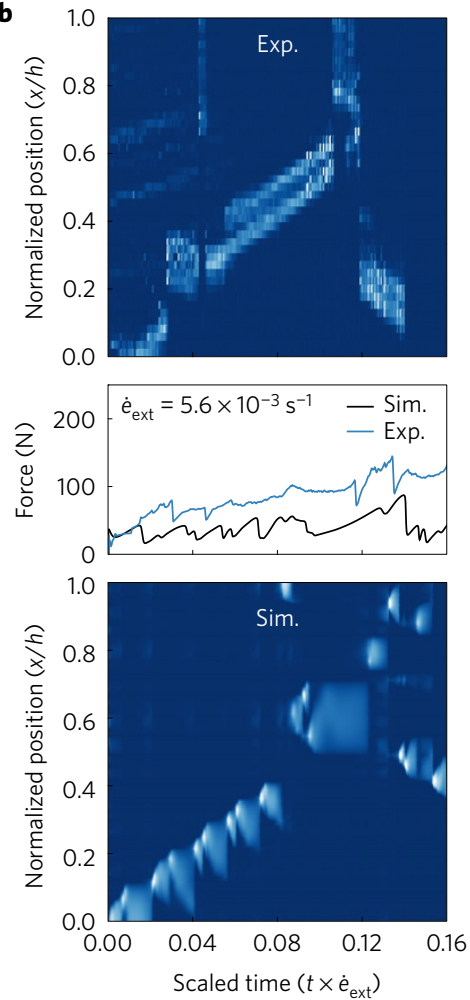
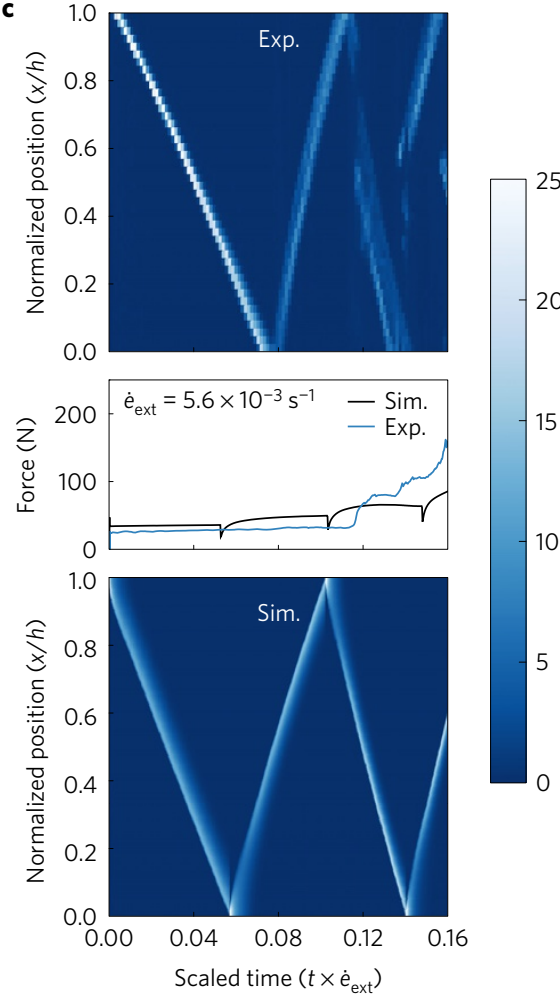

20

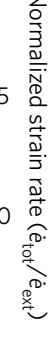

Figure 3 | Spacetime plots of deformation activity in natural snow. Snow density $\rho=275 \mathrm{~kg} \mathrm{~m}^{-3}$, deformation rate $\dot{e}_{\text {ext }}=5.6 \times 10^{-3} \mathrm{~s}^{-1}$. a,b, As-harvested samples. c, sieved sample. The top plots show the deformation activity as deduced experimentally from DIC, while the bottom plots show simulation data. The centre graphs are the corresponding force versus time/strain curves. The deformation of samples a and $\mathbf{c}$ is also shown in Supplementary Movies 3 and 4.

phenomena we use a phenomenological plasticity model, which we present here in a simplified, scalar version. The reader is referred to Supplementary Methods 2 for a derivation of the scalar equations from a fully tensorial constitutive model. Deformation is described in terms of the spatially homogeneous stress $s$, the plastic strain $e(x)$, which is a function of the axial position, and an internal variable $S$ which characterizes the structural strength contribution of intergranular bonds. This variable accounts, in a phenomenological manner, for the competing processes of bond breaking and bond recovery (sintering/ageing).

The stress is calculated from the plastic strain $e(x)$ and the strain $e_{\text {ext }}$ that is imposed by the downward motion of the piston according to

$$
s=E\left(e_{\mathrm{ext}}-\langle e\rangle\right)
$$

where \langle\rangle denotes the spatial average and $E$ is the Young's modulus of the sample. Equation (1) states a quasi-static relation between stress and strain: it neglects dynamic effects associated with elastic wave propagation. This is motivated by the observation that, with typical sound velocities in dense snow above $500 \mathrm{~m} \mathrm{~s}^{-1}$ (ref. 18), sound propagation times in our samples are below $5 \times 10^{-4} \mathrm{~s}-$ over four orders of magnitudes less than all other characteristic times in our experiments. By contrast, Guillard et al. ${ }^{19}$ relate propagating compaction bands in brittle porous materials such as cereals, which exhibit some remarkable similarities with the present observations, to viscoelastic waves. A detailed discussion of this issue is provided in Supplementary Methods 3.

The plastic strain rate is given by

$$
\partial_{t} e=\dot{e}_{0}\left(\frac{\Phi}{\sigma_{y}}\right)^{m} H(\Phi)
$$

where $m$ is the strain-rate exponent, $\dot{e}_{0}$ a prefactor with the dimension of a strain rate, and $H$ is Heaviside's function $(H(\phi)=1$ for $\Phi>0$ and $H=0$ otherwise). The yield stress $\sigma_{y}$ and yield function $\Phi$ depend on stress, strain, and on the internal variable $S$ according to

$$
\sigma_{y}=\sigma_{0}\left(\frac{1}{1-e / e_{c}}\right)^{b}(1+S), \quad \Phi=s-\sigma_{y}+\sigma_{0} \xi^{2} e_{x x}
$$

The yield stress $\sigma_{y}$ is a measure of the compressive strength of the material. It increases with increasing density (increasing compressive strain $e$ ) and diverges at a critical compressive strain $e_{c}$. This divergence is characterized by the exponent $b$, which for a cohesionless granulate corresponds to the jamming exponent. The factor $\sigma_{0}$ characterizes the strength of the unbonded $(S=0)$ and uncompacted $(e=0)$ granulate. The yield function $\Phi$ is evaluated as the difference between the compressive stress $s$ and the yield stress $\sigma_{y}$, corrected by a spatial coupling term with length scale $\xi$. This term increases the rate of plastic flow in locations where $e$ has a minimum $\left(e_{x x}>0\right)$ and decreases it in locations where $e$ has a maximum; it is thus of a diffusive nature.

Finally, the evolution of the internal variable $S$ is given by

$$
\partial_{t} S=-\left(\frac{\partial_{t} e}{\varepsilon_{S}}\right) S+\frac{1}{\tau}\left(S_{\infty}-S\right)
$$

This equation describes the competition between deformationinduced structural softening (first term on the right-hand side of equation (4), where $\varepsilon_{S}$ is the characteristic softening strain) and strength recovery by rapid sintering/ageing (second term on the right-hand side, with ageing time constant $\tau$ ).

Equations (1)-(4) define a set of integro-differential equations which we solve under the boundary condition that the plastic strain 
gradient $\partial_{x} e$ must vanish at the specimen ends $x=0$ and $x=h$. A detailed discussion of model parameters and initial conditions is found in Supplementary Methods 2. We use a common set of parameters for both natural and artificial snow samples, while accounting for differences in density by re-scaling the elastic constants and initial strength parameters by density-dependent factors (see Supplementary Table 2). The remaining differences in deformation behaviour between the different samples can be related to differences in specimen age, and in the degree of disorder of the snow microstructure. All simulations shown in Fig. 2 refer to specimens with age $t_{\mathrm{a}}=300 \mathrm{~s}$. For specimens of different age, we adjust the initial strength by an age-dependent factor such that $\sigma_{y}(t=0) \propto t_{a}^{0.2}$ (ref. 20). For saw-cut natural snow a high initial strength corresponding to an estimated age $t_{\mathrm{a}}=10^{6} \mathrm{~s}$ was used, whereas for the sieved samples we set $t_{\mathrm{a}}=300 \mathrm{~s}$, corresponding to the time between sieving and testing. Disorder of the snow microstructure is modelled by assigning statistically distributed initial strength values to the sites of our discretization grid of lattice constant $\xi$. For the strength distribution we use a Weibull distribution where, for artificial snow samples as well as for sieved natural snow, we assume a high Weibull exponent $\beta=10$ corresponding to fairly uniform local strength, whereas for saw-cut natural snow we use a low exponent $\beta=1$, which is in line with experimental investigations of the strength statistics of saw-cut natural snow samples reported by Kirchner and colleagues ${ }^{21}$. The simulations reproduce all essential features of the observed band propagation patterns and stress-strain curves. Nucleation of new bands is associated with stress oscillations. In homogeneous samples we consistently observe bouncing bands (see Figs $2 \mathrm{~b}$ and $3 \mathrm{c}$ ). Local strength fluctuations (low Weibull exponent) may induce band arrest and/or intermittent band propagation, as in Fig. 3a,b. Increasing the initial strength to reflect increased sample age leads to larger band strains and higher band propagation stresses, consistent with the experimental data (Supplementary Fig. 6).

Further experimental work will be needed to directly characterize the microstructural processes occurring in the compaction bands. To this end, in situ X-ray microtomography (XRMT) would be the method of choice. This method has been used to study microstructure evolution during compaction of solid foams (see, for example, ref. 22), where local structural softening (cell wall fracture or buckling) and compaction band formation are intrinsic features of the early stage of deformation ${ }^{23,24}$. XRMT has also been successfully used to study microstructure evolution during low-velocity deformation of snow ${ }^{25,26}$. However, currently accessible image acquisition rates do not allow for in situ monitoring of the propagating bands observed in our experiments, as band propagation times are typically of the order of a few minutes only. Advances in highthroughput XRMT may enable this in the foreseeable future. Even in the absence of in situ imaging possibilities, ex situ XRTM images taken before and after deformation may help, in conjunction with micro-scale mechanical modelling ${ }^{27}$, to parametrize macroscopic constitutive models and to further validate their predictions.

Data availability. The data that support the plots within this paper and other findings of this study are available from the corresponding author upon request.

\section{Received 14 July 2016; accepted 24 October 2016;} published online 21 November 2016

\section{References}

1. Zaiser, M. \& Hähner, P. A unified description of strain-rate softening instabilities. Mater. Sci. Eng. A 238, 399-406 (1997).

2. Narita, H. Mechanical behaviour and structure of snow under uniaxial tensile stress. J. Glaciol. 26, 275-282 (1980).

3. Lebyodkin, M., Dunin-Barkowskii, L., Brechet, Y., Estrin, Y. \& Kubin, L. P. Spatio-temporal dynamics of the Portevin-Le Chatelier effect: experiment and modelling. Acta Mater. 48, 2529-2541 (2000).
4. Rizzi, E. \& Hähner, P. On the Portevin-Le Chatelier effect: theoretical modeling and numerical results. Int. J. Plast. 20, 121-165 (2004).

5. Fielding, S. M. \& Olmsted, P. D. Spatiotemporal oscillations and rheochaos in a simple model of shear banding. Phys. Rev. Lett. 92, 084502 (2004).

6. Zaiser, M. \& Hähner, P. Oscillatory modes of plastic deformation - theoretical concepts. Phys. Status Solidi $b \mathbf{9 9}, 267-330$ (1997)

7. Wu, Q., Andreopoulos, Y., Xanthos, S. \& Weinbaum, S. Dynamic compression of highly compressible porous media with application to snow compaction. J. Fluid Mech. 542, 281-304 (2005).

8. Ella, S., Formagne, P.-Y., Koutsos, V. \& Blackford, J. R. Investigation of rubber friction on snow for tyres. Tribol. Int. 59, 292-301 (2013).

9. Schweizer, J. Review of dry snow slab avalanche release. Cold Reg. Sci. Technol. 30, 43-57 (1999).

10. McClung, D. M. Shear fracture precipitated by strain softening as a mechanism for dry slab avalanche release. J. Geophys. Res. 84, 3519-3526 (1979).

11. Birkeland, K. W., Kronholm, K., Logan, S. \& Schweizer, J. Field measurements of sintering after fracture of snowpack weak layers. Geophys. Res. Lett. 33, L03501 (2006).

12. Szabo, D. \& Schneebeli, M. Subsecond sintering of ice. Appl. Phys. Lett. 90, 151916 (2007)

13. Blackford, J. R. Sintering and microstructure of ice: a review. J. Phys. D 40, R355-R385 (2007).

14. De Montmollin, V. Shear tests on snow explained by fast metamorphism. J. Glaciol. 28, 187-198 (1982).

15. Kinosita, S. Compression of snow at constant speed. Phys. Snow Ice Proc. 2, 911-927 (1967)

16. Louchet, F. Creep instability of the weak layer and natural slab avalanche triggerings. Cold Reg. Sci. Technol. 33, 141-146 (2001).

17. Reiweger, I., Schweizer, J., Dual, J. \& Herrmann, H. J. Modelling snow failure with a fibre bundle model. J. Glaciol. 55, 997-1002 (2009).

18. Reiweger, I., Mayer, K., Steiner, K., Dual, J. \& Schweizer, J. Measuring and localizing acoustic emission events in snow prior to fracture. Cold Reg. Sci. Technol. 110, 160-169 (2015).

19. Guillard, F., Angolan, P., Shen, L., Valdes, J. R. \& Einav, I. Dynamic patterns of compaction in brittle porous media. Nat. Phys. 11, 835-838 (2015).

20. Podolskiy, E. A. et al. Healing of snow surface-to-surface contacts by isothermal sintering. Cryosphere 8, 1651-1659 (2014).

21. Kirchner, H. O. K., Peterlik, H. \& Michot, G. Size independence of the strength of snow. Phys. Rev. E 69, 011306 (2004)

22. McDonald, S. A., Mummery, P. M., Johnson, G. \& Withers, P. J. Characterization of the three-dimensional structure of a metallic foam during compressive deformation. J. Microsc. 223, 150-158 (2006).

23. Forest, S., Blazy, J.-S., Chastel, Y. \& Moussy, F. Continuum modeling of strain localization phenomena in metallic foams. J. Mater. Sci. 48, 5903-5910 (2005).

24. Zaiser, M., Mill, F., Konstantinidis, A. \& Aifantis, K. E. Strain localization and strain propagation in collapsible solid foams. Mater. Sci. Eng. A 567, 38-45 (2013).

25. Wang, X. \& Baker, I. Observation of the microstructural evolution of snow under uniaxial compression using X-ray computed microtomography. J. Geophys. Res. 118, 12371-12382 (2013).

26. Schleef, S., Löwe, H. \& Schneebeli, M. Hot-pressure sintering of low-density snow analyzed by X-ray microtomography and in situ microcompression. Acta Mater. 71, 185-194 (2014)

27. Hagenmüller, P., Theile, T. C. \& Schneebeli, M. Numerical simulation of microstructural damage and tensile strength of snow. Geophys. Res. Lett. 41, 86-89 (2014).

\section{Acknowledgements}

T.W.B. acknowledges financial support by EPSRC - DTP. S.L., S.S., G.W. and M.Z. acknowledge computational support by ZISC.

\section{Author contributions}

T.W.B., T.J.S. and J.R.B. designed the apparatus and DIC imaging system, T.W.B. carried out the experiments, S.S. and S.L. analysed the DIC data, G.W., S.S. and S.L. wrote the simulation code and performed simulations, M.Z. formulated and parametrized the model, performed simulations and wrote the manuscript. All authors were involved in editing the manuscript.

\section{Additional information}

Supplementary information is available in the online version of the paper. Reprints and permissions information is available online at www.nature.com/reprints. Correspondence and requests for materials should be addressed to M.Z.

\section{Competing financial interests}

The authors declare no competing financial interests. 